\title{
Empirical Interconnect Crosstalk Characterization for High Level Synthesis
}

\author{
A. A. de Carvalho \\ Department of Information and Computer Science \\ University of California, Irvine, CA 92697-3425, USA \\ antenor@ics.uci.edu \\ phone: (714)824-8168 fax: (714) 824-4056

\section{F. J. Kurdahi} \\ Department of Computer Engineering, \\ University of California, Irvine, CA 92697, U.S.A. \\ kurdahi@ece.uci.edu \\ phone: (714)824-8104 fax:(714) 824-2321
}

\begin{abstract}
As device geometries continue to get smaller delay and fault specification for deep-submicron technologies is evolving into a quite complicated task. The cumulative effect of gate delays are now less important than the interconnect delays, particularly in chip level interconnections. Potential crosstalk effects must also be taken into account. In this paper we concentrate on the crosstalk effects between neighboring wires in deep submicron interconnection structures and its characterization for use in higher levels of the design hierarchy. Realistic models were used and HSPICE simulation results are presented. Empirical generalization between experimental data and estimated crosstalk potential is performed.
\end{abstract}

\section{Keywords}

crosstalk, deep submicron, estimation, interconnect templates 


\section{INTRODUCTION}

The expression "deep submicron" usually refers to technologies in which the smaller geometric features are sized below $0.6 \mu \mathrm{m}$, i.e., in these technologies the lithographical mask generation stage of the fabrication process has enough resolution to generate layout primitives whose minimum dimensions are below 0.6 $\mu \mathrm{m}$.

It has been found that the physical characteristics of these technologies considerably complicate the design process (Watts, 1989). Although the logic gate delays have been substantially reduced because the transistor capacitances inside the gate cells are smaller, the interconnection delays have increased in relation with the gate delays. This is due to the fact that wires with smaller cross section naturally have bigger resistance. Even though the area capacitances tend to be smaller in sub-micron technology the closeness between wires tends to increase inter-wire capacitances.

Furthermore, the crosstalk interaction between different wires in buses, for example, or between wires in different layout layers also influence the way the data is transferred within the chip. Since device geometries have shrunk, so have the minimum distance between adjacent conductors, increasing the interaction among those conductors by means of stray capacitances, resistance and inductance. Glitches are more likely to occur in these conditions and therefore fault analysis strategies must be implemented. Timing optimization schemes that do not take into consideration these effects can fail to generate a working chip at the first design iteration, forcing the design team to reassess its decisions in the earlier stages of design. The main consequence is, of course, a longer design cycle time with subsequent delay in reaching the market before the other competitors, with obvious loss of revenue.

\section{BACKGROUND}

Several techniques have been proposed in the literature for the analysis of VLSI interconnects and coupled microstrip lines (Rubio ,1994), (Zhang, 1992), (Liaud, 1994), (Xie, 1993), (Achar, 1995), (Eo, 1995). The estimation of area and timing has been addressed by various papers (Ramachandran, 1994), (Ramachandran, 1992), (Kaptanoglu,1996) as well as the linking between physical and higher abstraction levels (Jha, 1994), (Kurdahi, 1993), (Mintz, 1994). A better characterization of crosstalk, delay and transmission line behavior in interconnect wiring is necessary to reduce the hiatus between estimated timing and the final physical implementation (Rabaey, 1996). In the following item we present some background information about interconnect behavior. 


\section{Interconnect Delay}

The increase in the number of gates and RTL components in a single chip indicates that the average interconnect length also increases. The wiring length $L$ for an average net in a circuit with area $A$ can be approximated by

$$
L=\frac{\sqrt{\pi}}{5} \sqrt{A}=\frac{\sqrt{A}}{3}
$$

There are some conclusions that can be drawn if there is a function in which $L$ increases with $A$. The resistance and capacitance of a line are all proportional to wire length (Rabaey, 1996) which in turn is proportional to $A$. In addition, signals have to travel larger distances because the die sizes are getting larger and delays and crosstalk associated with transmission line effects also tend to get larger (Watts, 1989), (Sorkin, 1987).

\section{DESIGN METHODOLOGIES IN DEEP SUBMICRON DESIGN}

Design methodologies are expected to change in view of the greater importance and influence the physical level has in the logic and behavioral levels. The synthesis of a chip based on early design exploration must now take into account the topological and lossy characteristics of buses.

\section{Traditional design synthesis}

Traditional design synthesis methodologies are those which do not take primary care of the interconnect impact in the timing analysis and logic optimization. In these tools the primary contributors of delay in any logical path in the design are assumed to be the gates, not the wires or interconnects. This methodology consists primarily of the stages described in Gajski (1994)

After high-level synthesis a register transfer level (RTL) netlist is obtained as well as boolean functions representing each of the RTL components. This netlist serves as one input to the logic synthesis stage. During the logic synthesis the input is converted into a netlist of gates which are then placed in a floorplan and routed. Physical characteristics of the circuit are extracted from the layout and then the circuit is simulated again with enhanced physical and delay information. If the simulation indicates that the design is working reliably for a set of timing and area constraints then the layout can be sent to the foundry. If not, the designers must reevaluate the circuit at the logic level and modify some of the critical paths to meet the timing and/or area constraints. The design may not converge after several iterations in the cycle described above. In this case the weight of the physical delay information is of such influence in the back-annotated simulations that the design usually does not meet all the timing constraints. 


\section{Deep submicron design considerations}

The design process described above has a fundamental flaw: it does not take detailed interconnect information into account when doing scheduling, allocation and binding. The high-level synthesis process is, therefore, void of appropriate data concerning bus data transfers and wiring delay in general. One way of obtaining some physical information in an early level of design is to perform floorplan estimation. The floorplan estimator should be able to feedback some interconnect information to the high-level synthesis stage. In this case we would end with a set of tools like the one in Figure 1.

This way significant wiring delay can be considered in the front end and used as any of the other elements such as functional units, storage etc. Thus, we need a suitable high-level interconnect model characterized as a function of its delay, area, bit-width and crosstalk. The following items present some basic aspects of deep submicron interconnects.

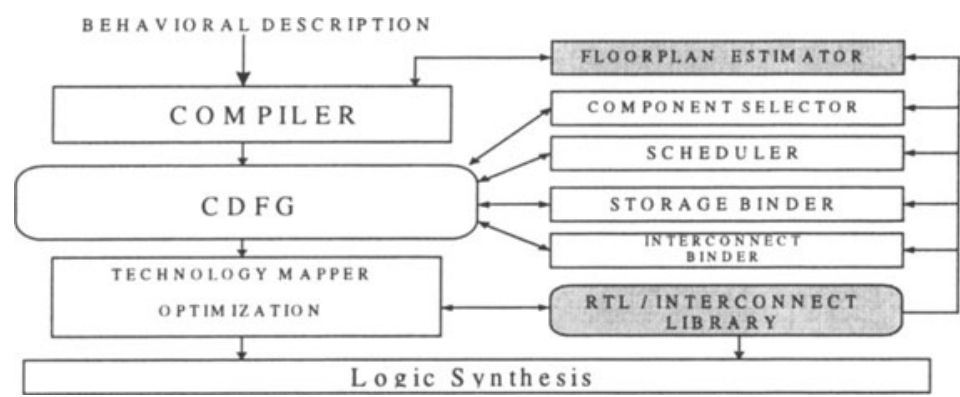

Figure 1 High-level synthesis system with enhanced interconnect information

\section{Interconnect behavior}

The delay of a bus wire can be considered to be dependent on its capacitance, resistance and inductance. Each of these three parameters have effects on the interconnect behavior because they may induce noise, like crosstalk, which reduces the reliability and fault-tolerance of the circuit, and increase the propagation delay.

The propagation delay is proportional to the wire capacitance $\mathrm{C}_{\mathrm{int}}$ as expressed by

$$
\begin{aligned}
& T_{p}=\frac{C_{i n t}}{2 V_{d d}}\left(\frac{1}{\beta_{n}}+\frac{1}{\beta_{p}}\right) \\
& C_{i n t}=e_{o x} L\left[\left(\frac{W}{t_{o x}}\right)+0.77+1.06\left(\frac{W}{t_{o x}}\right)^{0.25}+1.06\left(\frac{H}{T_{o x}}\right)^{0.5}\right]
\end{aligned}
$$


where $L$ is the wire length, $W$ is the wire width, $H$ is the wire thickness, $t_{o x}$ is the oxide thickness, $e_{o x}$ is the permittivity of the oxide and $\beta_{n}$ and $\beta_{p}$ are the betas for the $\mathrm{N}$ and $\mathrm{P}$ transistors respectively. The above equation includes fringing capacitances (Rabaey, 1996). The interconnect resistance can be expressed by:

$$
R=(\text { rho } . L) /(H . W)
$$

where rho, $L, H$ and $W$ are the conductor's resistivity, lenght, height and width.

Interconnect wires exhibit inductive parasitics as present in bonding wires and chip packages. A changing current propagating through an inductor generates a voltage drop. The magnitude of the voltage drop $(\Delta V)$ for an inductance $L$ and change in current $(\delta I / \delta t)$ is:

$\Delta V=L(\delta I / \delta t)$

The wave propagation equation in a lossy transmission line can be expressed by

$$
\frac{\partial^{2} v}{\partial x^{2}}=r c \frac{\partial v}{\partial t}+l c \frac{\partial^{2} v}{\delta t^{2}}
$$

as shown in (Rabaey, 1996).

\section{Crosstalk factor}

Crosstalk is caused by capacitive and inductive coupling between neighboring interconnect lines. Another source of crosstalk among circuits is due to common impedance in ground loops. The crosstalk between different lines in a bus must be taken into account in order to preserve data integrity throughout the synthesis process. It has been shown [1] that circuit wires coupled by parasitic capacitances can reach a level of interference high enough to affect the signal reliability, i.e., the voltage level variations in neighboring wires can affect each other to the point in which the logic information in these wires is changed. Therefore, crosstalk must be seen as a potential fault condition in submicron IC's.

Crosstalk coupling is defined as the ratio of the power in a disturbing line to the induced power in the disturbed line. Consider two bus lines adjacent to each other, Line 1 and Line2. The amount of crosstalk between them is expressed in $\mathrm{dB}$ by:

$$
\text { Crosstalk }(\text { line } 1, \text { line } 2)=10 \log (P 2 / P 1)=20 \log (V 2 / V 1)
$$

where $\mathrm{V} 1$ is the voltage in the disturbing wire and V2 is the voltage in the disturbed wire as induced by capacitive, inductive and resistive coupling. 
Based on expression (1) we can define a crosstalk factor Crosstalk Factor $_{\text {in }}$ (Line1,Line2) $=f\left(\Delta V_{2}, \Delta V_{1}\right)$ in which the interference between line 1 and line 2 is expressed by the difference in their amplitude module:

Crosstalk $_{\text {Facior }}($ line1, line 2$)=20 \log \left(V 2_{\text {up }}-V 2_{\text {down }} / V 1_{\text {up }}-V 1_{\text {down }}\right)$

where $\mathrm{V} 1_{\mathrm{up}}$ is the maximum voltage for line $1, \mathrm{~V} 1_{\text {down }}$ is the minimum voltage for line $1, \mathrm{~V} 2_{\mathrm{up}}$ is the maximum induced voltage for line 2 and $\mathrm{v} 2_{\text {down }}$ is the minimum induced voltage for line 2 . The crosstalk factor,also expressed in $\mathrm{dB}$ 's, has negative values, indicating the atennuation between wires.

In order to characterize the crosstalk potential for high level interconnect descriptions we assume that the crosstalk is proportional to the thickness of the conductor, the length of the interconnect wires and inversely proportional to the spacing between conductors. A simple empirical expression for the crosstalk potential is

Crosstalk $_{\text {potential }}=\mathrm{K}\left(L / L_{\text {safe }}\right) \cdot(T H / S P)$

where $L$ is the wire length, $L_{\text {safe }}$ is the length considered to be ideal for crosstalk avoidance, $T H$ is the wire thickness, $S P$ is the spacing between conductors and $K$ is a constant related with the technology being used and $B$ is the linear coefficient for the interpolated function obtained from experimental data. Using this simple expression we can classify interconnect buses regarding their potential for crosstalk interference.

Our objective is to find a function Crosstalk $_{\text {estimate }}=f\left(\right.$ Crosstalk $\left._{\text {potential }}\right)$ that approximates the actual experimental data as calculated in (8):

Crosstalk $_{\text {estimate }}=f\left(\right.$ Crosstalk $\left._{\text {potential }}\right) \approx$ Crosstalk $_{\text {Factor }}$

\section{EXPERIMENTAL RESULTS}

We address the crosstalk in two parallel lines using the following technologies: 0.6 $\mu \mathrm{m}$ and $0.25 \mu \mathrm{m}$. The circuit topology is shown in Figure 2. The circuit was simulated using Hspice level 3 transmission lines and extracted Hspice netlists from Compass Tools standard cell libraries. The cells were extracted from layout using the Extract 2 tool and then converted to Hspice netlists using the Netlist 
utility. The transistor models used in Hspice were of level 13. In out first example, both lines are active, driven by pulse voltage sources of varying frequencies. After a certain time, the tri-state buffer is disabled so that the output of the buffer connected to the input of the disturbed transmission line goes into a high impedance state. The crosstalk then is observed between those lines.

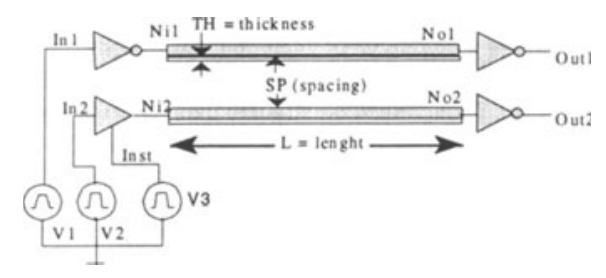

Inverters: $1 \mathrm{X}$

Tri-state buffer: $1 \mathrm{X}$

transmission line lossy model

in sea of dielectric

transistor MOS level 13

Figure 2 Test Hspice circuit with subcircuit and model descriptions.

By modeling the source and destination impedances as detailed extracted gate of an actual library the simulation results are more realistic than those observed in other references where the drivers and load are usually modeled as a pair resistor-capacitor.

The simulations take into account three varying geometric parameters: wire length, thickness and spacing. In the first batch of results we have three different line lengths $(500 \mu \mathrm{m}, 1000 \mu \mathrm{m}$ and $1500 \mu \mathrm{m})$ and two thicknesses $(1 \mu \mathrm{m}$ and $2 \mu \mathrm{m})$ for a $0.6 \mu \mathrm{m}$ process. The simulation results, shown in Table 1 clearly show that the greater the thickness, the worse the crosstalk. In all cases we used expression (8). For a $0.25 \mu \mathrm{m}$ process the results are shown in table 2 . These results show that smaller feature size technologies are indeed prone to hazardous interconnect crosstalk, as previously stated.

Of particular importance is the behavior of the high impedance wire. If the tri-state buffer's output is switched into high-impedance when the line itself is in transition from one logic level to another, the average level of disturbance can fall exactly in

Table 1 Crosstalk Factor $(\mathrm{dB})$ for $0.6 \mu \mathrm{m}$ technology

\begin{tabular}{|c|c|c|c|c|c|c|c|c|c|}
\hline \multirow{3}{*}{$\frac{L(\mu m)}{T H(\mu m)} \frac{S P(\mu m)}{S(n)}$} & \multicolumn{3}{|c|}{500} & \multicolumn{3}{|c|}{1000} & \multicolumn{3}{|c|}{1500} \\
\hline & \multicolumn{2}{|c|}{1} & \multirow{2}{*}{$\frac{2}{1.8}$} & \multicolumn{2}{|c|}{1} & \multirow{2}{*}{$\frac{2}{1.8}$} & \multicolumn{2}{|c|}{1} & \multirow{2}{*}{$\begin{array}{c}2 \\
1.8\end{array}$} \\
\hline & 1.8 & 3.6 & & 1.8 & 3.6 & & 1.8 & 3.6 & \\
\hline & -15.1 & -17.9 & -14.1 & -15.3 & -19.5 & -12.5 & -15.2 & -19.8 & -13.2 \\
\hline
\end{tabular}


Table 2 Crosstalk Factor (dB) for $0.25 \mu \mathrm{m}$ technology

\begin{tabular}{|c|c|c|c|c|c|c|c|c|c|}
\hline \multirow{3}{*}{$\frac{\frac{L(\mu m)}{T H(\mu m)}}{S P(\mu m)}$} & \multicolumn{3}{|c|}{500} & \multicolumn{3}{|c|}{1000} & \multicolumn{3}{|c|}{1500} \\
\hline & \multicolumn{2}{|c|}{0.75} & \multirow{2}{*}{$\frac{1.5}{0.75}$} & \multicolumn{2}{|c|}{0.75} & \multirow{2}{*}{$\frac{1.5}{0.75}$} & \multicolumn{2}{|c|}{0.75} & 1.5 \\
\hline & 0.75 & 1.5 & & 0.75 & 1.50 & & 0.75 & 1.5 & 0.75 \\
\hline & -8.6 & -13.2 & -6.8 & -8.2 & -11.7 & -6.2 & -7.4 & -11.4 & -5.82 \\
\hline
\end{tabular}

the threshold region of the destination gate (in our case an inverter), causing erratic behavior in its output due to crosstalk as shown in Figure 3. It should be noted that one of the functions of the output gate would be to restore the signal arriving from the interconnect to a strong and defined logic level. In the simulation results all of the three interconnect lengths have similar crosstalk factors but the only one not to have the output significantly disturbed is the $500 \mu \mathrm{m}$ wire. The other two show severe voltage level disturbances, even after the destination gate output. We also performed simulations using with one of the wires in "quiet" mode, i.e., the disturbing wire is switched while the disturbed wire is set at a constant voltage level in its input, in our case 0 volts. In this case the voltage sources in Figure 2, V2 and V3, were set to DC operation at 0 and 3.3 volts, respectively. The simulations, as expected, show both the influence of wire thickness and spacing.

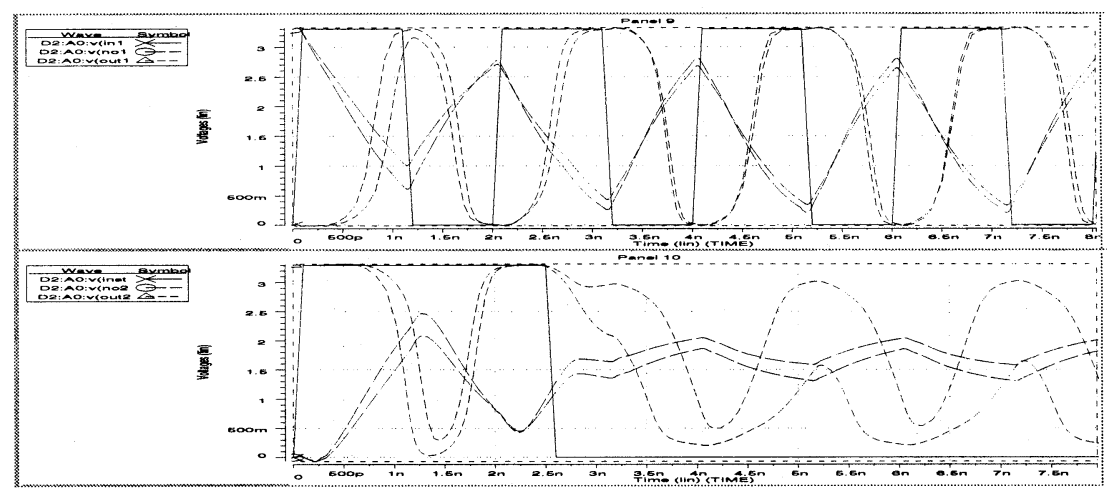

Figure 3 Simulation results for $0.6 \mu \mathrm{m}$ technology with $\mathrm{SP}=1.8 \mu \mathrm{m}, \mathrm{TH}=1$ and 2 $\mu \mathrm{m} \mathrm{L}=1500 \mu \mathrm{m}$. The top window has the waveforms for signals in the "active" wire and the bottom window has waveforms for the "passive" wire. After $2.5 \mathrm{~ns}$ the tri-state buffer is deactivated and the the wire goes into high impedance. N02 and OUT2 are indicated in Figure 2. 


\section{Crosstalk rating}

Since our objective is extracting enough information to be used in higher levels of design without jeopardizing the main advantages of higher levels of abstraction, i.e., faster design exploration, we use some of the data obtained during this simulation to create a sample of interconnection elements with additional crosstalk information.

By organizing the interconnect crosstalk ratings as in table 5 we can see the relation

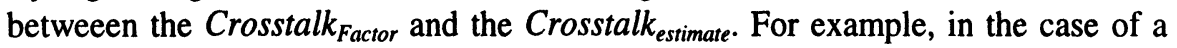
$0.25 \mu \mathrm{m}$ technology, for a length $\mathrm{L}=500 \mu \mathrm{m}$, and $\mathrm{L}_{\text {safe }}=500 \mu \mathrm{m}, \mathrm{TH}=0.75 \mu \mathrm{m}$, $\mathrm{SP}=1.5 \mu \mathrm{m}$ we would have the following expression for the crosstalk potential:

Crosstalk $_{\text {Potential }}=\left(\mathrm{L} / \mathrm{L}_{\text {safe }}\right)(\mathrm{TH} / \mathrm{SP})=\mathrm{TH} / \mathrm{SP}=0.5$

As a first approximation we could use an expression of the form $y=k \cdot x^{-c}$ to characterize the crosstalk factor Crosstalk factor as a function of the crosstalk potential Crosstalk ${ }_{\text {Potennial }}$ Let $x=$ crosstalk $_{\text {potential }}, k$ a constant which depends on the experimental data and $y$ the crosstalk factor Crosstalk esimate . From algebraic manipulations we find that, for this particular technology, $c=0.208$ and $k=14.9$.

Therefore, we have:

$\mathrm{Y}=\mathrm{K}(\mathrm{X})^{-\mathrm{c}}$

if $Y=$ Crosstalk $_{\text {estimate }}$ and $X=$ Crosstalk $_{\text {potential }}=\left(L / L_{\text {safe }}\right)(T H / S P)$ then

Crosstalk $_{\text {estimate }}=K\left(\left(L / L_{\text {safe }}\right)(T H / S P)\right)^{-c}$

or

Crosstalk $_{\text {estimate }}=14.9(T H / S P)^{-0.208}$

since $\mathrm{L}=\mathrm{L}_{\text {sare }}$ in this case, with some of the points shown in table 5 for $T H=0.75$ and $1.5 \mu \mathrm{m}$, and $S P=0.75$ and $1.5 \mu \mathrm{m}$. The above expression is a function of the crosstalk potential and was extracted for this technology by interpolating the experimental data.

In Table 5 we show the relation between the estimated crosstalk (Crosstalk ${ }_{\text {esinate }}$ ) as defined in (11) and the experimental data, expressed by the module of the Crosstalk ${ }_{\text {Factor }}$, as defined in (8). By analyzing this table, we notice that the estimated data has good correlation with the HSPICE simulation results. 
Table 5 Crosstalk estimate vs ICrosstalk factorl (measured data) (dB)

\begin{tabular}{|c|c|c|c|c|c|}
\hline \multicolumn{2}{|c|}{$L=500 \mu \mathrm{m}$} & \multicolumn{2}{|c|}{$L=1000 \mu \mathrm{m}$} & \multicolumn{2}{|c|}{$L=1500 \mu \mathrm{m}$} \\
\hline $\begin{array}{l}\text { Crosstalk } \\
\text { Estimate }\end{array}$ & $\begin{array}{c}\text { | Crosstalk } \\
\text { factor I }\end{array}$ & $\begin{array}{l}\text { Crosstalk } \\
\text { Estimate }\end{array}$ & $\begin{array}{c}\text { I Crosstalk } \\
\text { factor I }\end{array}$ & $\begin{array}{l}\text { Crosstalk } \\
\text { Estimate }\end{array}$ & $\begin{array}{c}\text { | Crosstalk } \\
\text { factor I }\end{array}$ \\
\hline 17.21078 & 20 & 23.65006 & 20.4 & 26.39016 & 20.8 \\
\hline 14.9 & 14.9 & 18.3 & 18.3 & 20 & 19 \\
\hline 13.69491 & 13.9 & 15.75061 & 16.2 & 17.00566 & 17.8 \\
\hline 12.89947 & 12.9 & 14.16022 & 14.1 & 15.15717 & 16.2 \\
\hline 12.31444 & 11.9 & 13.03807 & 13.45 & 13.8629 & 14.7 \\
\hline 11.85619 & 10.9 & 12.18754 & 12.8 & 12.88788 & 13.3 \\
\hline 11.16755 & 8.8 & 10.95693 & 11.5 & 11.48698 & 12 \\
\hline 10.26433 & 4.9 & 9.430509 & 8.9 & 9.767187 & 9.8 \\
\hline
\end{tabular}

This approach, which takes into consideration physical process information, can perform a good estimation of the crosstalk effects in the technologies used here.

This is one stage in an ongoing research effort aimed at characterizing interconnect behavior for high-level synthesis system by means of interconnect templates. Some of these interconnection modules would use this information in the following manner:

Component = IT (delay, shape, crosstalk $\left._{\text {estimate }}\right)$

where "IT" stands for Interconnect Template", delay is as defined in expression (2), shape is the size of the interconnect bus which is a function of wire width, spacing and the number of wires, and crosstalk is the rating defined as in (11). IT's are the subject of an upcoming paper. More detailed experimental data can be found in the unabridged version of this paper in http://jblevins.ics.uci.edu/. 


\section{CONCLUSIONS}

In this paper we presented an empirical model for high level classification of interconnects. Our approach is to create an interconnect component library with interconnect cells classified, or rated, according to their physical behavior regarding crosstalk, delay and shape. These interconnect cells are to be used in higher levels of the design cycle so as to improve its convergence and therefore shorten its turn around time. In other to give an example of this rating we build a sample library of interconnect components based on $0.6 \mu \mathrm{m}$ and $0.25 \mu \mathrm{m}$ technologies.

\section{REFERENCES}

Achar, R. et al (1995) Simulation of Delay and Crosstalk in High Speed VLSI Interconnects, in Proceedings 1995 Canadian Conference on Electrical and Computer Engineering (ed. Gagnon), IEEE.

Eo Y. et al. (1995) Simulations and measurements of picosecond signal transients, propagation, and crosstalk on lossy VLSI interconnect. IEEE Transactions on Components, Packaging, and Manufacturing Technology, Part A, vol.18.

Gajski et al. (1994) Specification and Design of Embedded Systems. P T R Prentice Hall.

Hspice User's Manual, volume III, Meta-Software 1996.

Itazaki, N. et al.(1996) A fault simulation method for crosstalk faults in synchronous sequential circuits, in Proceedings of the Twenty-Sixth International Symposium on Fault-Tolerant Computing, IEEE Comput. Soc. Press.

Tang, T.K. and Nakhla, M.S. (1992) Analysis of high-speed VLSI interconnects using the asymptotic waveform evaluation technique. IEEE Transactions on Computer-Aided Design of Integrated Circuits and Systems, vol.11, (no.3):341-52.

Jha, P.K.; Ramachandran, C.; Dutt, N.D.; Kurdahi, F.J., (1994) An empirical study on the effects of physical design high-level synthesis, in Proceedings of the Seventh International Conference on VLSI Design.

Kurdahi, F.J.; Gajski, D.D.; Ramachandran, C.; Chaiyakul, V. (1993) Linking Register-transfer and Physical Levels of Design. IEICE Transactions on Information and Systems, vol.E76-D, (no.9):991-1005.

Kaptanoglu, S. (1996) Fast and accurate delay estimation for use in timing driven layout for FPGAs with segmented channels, in Proceedings of Custom Integrated Circuits Conference.

Liaud, A. et al. (1994) On Crosstalk Fault Detection in Hierarchical VLSI Logic Circuits, in Proceedings of IEEE $3^{r d}$ Asian Test Symposium, IEEE Comput. Soc. Press, 1994. 
Mintz, D. and Dangelo, C. (1994) Timing estimation for behavioral descriptions in Proceedings of the Seventh International Symposium on High-Level Synthesis. Rabaey, J. (1996) Digital Integrated Circuits: A Design Perspective. Prentice Hall. Ramachandran, C. (1992) Combined topological and functionality based delay estimation using at layout-driven approach for high level applications, in EURO-DAC '92. European Design Automation Conference.

Ramachandran, C. and Kurdahi, F.J.; Gajski, D.D.; Wu, A.C.-H. (1992) Accurate layout area and delay modeling for system level design, in IEEE/ACM International Conference on Computer-Aided Design.

Ramachandran, C. and Kurdahi, F.J. (1994) Combined topological and functionality-based delay estimation using a layout-driven approach for high-level applications. IEEE Transactions on Computer-Aided Design of Integrated Circuits and Systems, Dec. 1994.

Rubio, A. et al. (1994) An Approach to the analysis and Detection of Crosstalk Faults in Digital VLSI Circuits. IEEE Transactions on Computer-Aided Design of Intergrated Circuits and Systems, Vol.13, No. 3.

Sorkin, G. (1987) Assimptotically Perfect Trivial Global Routing: A Stochastic Analysis" IEEE Transactions On Computer-aided Design, vol. CAD-6, p. 820.

Watts, R.K. (1989) Submicron integrated circuits (ed. Wiley).

Xie, D. et al. (1993) Delay and Crosstalk Simulation of High-Speed VLSI Interconnects with Nonlinear Terminations. IEEE Transactions on Computeraided Design of Integrated Circuits and Systems, Vol. 12, no. 11.

Zhang, Q. et al. (1992) Minimization of Delay and Crosstalk in High-Speed VLSI Interconnects. IEEE Transactions on Microwave Theory and Techniques, Vol. 40 , no.7.

Antenor A. de Carvalho received the B.Sc. degree in electrical engineering and M. Sc. degree in Systems Engineering from the Federal University of Rio de Janeiro, Brazil, and a M. Sc. degree in Computer Science from the University of California in Irvine in 1991 and 1996 respectively. Between 1987 and 1991 he worked in the areas of computer aided design in Embratel and AD-Rio, and as consultant for private companies and government agencies in Brazil. Mr. Carvalho is currently working towards his Ph.D. degree in Computer Science at ICS/UCI, and his research interests are high-level synthesis, interconnect estimation and design methodologies for deep submicron VLSI design. Mr. Carvalho is a student member of ACM.

Fadi J. Kurdahi received the Bachelor of Engineering degree in Electrical Engineering from the American University of Beirut, Beirut, Lebanon in 1981, and the the M.S. degree in Electrical Engineering and the Ph.D. degree in Computer Engineering from the University of Southern California, Los Angeles, CA, in 1982 and 1987, respectively. His research interests are in high-level synthesis, estimation, and design methodology of large scale systems. Since 1987, he is with the ECE department at the University of California, Irvine. Dr. Kurdahi is a member of IEEE and ACM . 